PROCEEDINGS OF THE

AMERICAN MATHEMATICAL SOCIETY

Volume 135, Number 1, January 2007, Pages 229-236

S 0002-9939(06)08641-2

Article electronically published on June 29, 2006

\title{
PROPERTIES OF FIXED POINT SETS AND A CHARACTERIZATION OF THE BALL IN $\mathbb{C}^{n}$
}

\author{
BUMA L. FRIDMAN AND DAOWEI MA
}

(Communicated by Mei-Chi Shaw)

\begin{abstract}
We study the fixed point sets of holomorphic self-maps of a bounded domain in $\mathbb{C}^{n}$. Specifically we investigate the least number of fixed points in general position in the domain that forces any automorphism (or endomorphism) to be the identity. We have discovered that in terms of this number one can give the necessary and sufficient condition for the domain to be biholomorphic to the unit ball. Other theorems and examples generalize and complement previous results in this area, especially the recent work of Jean-Pierre Vigué.
\end{abstract}

\section{INTRODUCTION}

Let $D \subset \mathbb{C}^{n}$ be a bounded domain. Below we consider two families of self-maps of $D$. The first is the group $\operatorname{Aut}(D)$ of holomorphic automorphisms of $D$; the second is the set $H(D, D)$ of all holomorphic maps from $D$ to $D$, i.e., the set of endomorphisms of $D$.

Definition 0.1. A set $K \subset D$ is called a determining subset of $D$ with respect to $A u t(D)$ (or $H(D, D)$ resp.) if, whenever $g$ is an automorphism (resp. endomorphism) of $D$ such that $g(k)=k \forall k \in K$, then $g$ is the identity map of $D$.

The notion of a determining set was introduced earlier in a paper we wrote with our collaborators Steven G. Krantz and Kang-Tae Kim [5]. In that paper we attempted to find a higher-dimensional analog of the following result of classical function theory ([4, 17], 19, 20]): if $f: M \rightarrow M$ is a conformal self-mapping of a plane domain $M$ which fixes three distinct points, then $f(\zeta)=\zeta$.

Determining sets have been further investigated in the papers [6], 12], 21, [22.

Let $W_{s}(D)$ denote the set of $s$-tuples $\left(x_{1}, \ldots, x_{s}\right)$, where $x_{j} \in D$, such that $\left\{x_{1}, \ldots, x_{s}\right\}$ is a determining set with respect to $\operatorname{Aut}(D)$. Similarly, $\widehat{W}_{s}(D)$ denotes the set of $s$-tuples $\left(x_{1}, \ldots, x_{s}\right)$ such that $\left\{x_{1}, \ldots, x_{s}\right\}$ is a determining set with respect to $H(D, D)$. So $\widehat{W}_{s}(D) \subseteq W_{s}(D) \subseteq D^{s}$. We now introduce two numbers $s_{0}(D)$ and $\widehat{s}_{0}(D)$. In the case $\operatorname{Aut}(D)=i d, s_{0}(D)=0$, otherwise $s_{0}(D)$ is the least integer $s$, such that $W_{s}(D) \neq \emptyset$. The symbol $\widehat{s}_{0}(D)$ denotes the least integer $s$ such that $\widehat{W}_{s}(D) \neq \emptyset$. Hence, $s_{0}(D) \leq \widehat{s}_{0}(D)$.

In [5] we proved the inequality $s_{0}(D) \leq n+1$ for many (but not all) bounded domains in $\mathbb{C}^{n}$. In 21] J.-P. Vigué, using a different method, proved this estimate

Received by the editors August 2, 2005.

2000 Mathematics Subject Classification. Primary 32M05, 54H15. 
for all bounded domains in $\mathbb{C}^{n}$. Furthermore in 22 Vigué proved the estimate $\widehat{s}_{0}(D) \leq n+1$ for all bounded domains in $\mathbb{C}^{n}$.

Both estimates are the best possible, since for the unit ball $B^{n} \subset \mathbb{C}^{n}, s_{0}\left(B^{n}\right)=$ $n+1$. In section 1 we prove that the reverse is true: if $s_{0}(D)=n+1$ for a bounded domain $D \subset \mathbb{C}^{n}$, then $D$ is biholomorphic to the unit ball $B^{n}$. Obviously, $s_{0}(D)$ depends on how large $\operatorname{Aut}(D)$ is: for a smaller group, we expect a lower $s_{0}(D)$. This relationship is reflected below in Corollary 1.7.

If a positive integer $s \geq s_{0}(D)$, then $W_{s}(D) \neq \emptyset$, so there are $s$ points such that if an automorphism of $D$ fixes these points, it will fix any point of $D$. Now the question arises of whether the choice of these $s$ points is generic. To make it more precise we need to find out if $W_{s}(D)$ is open and everywhere dense in $D^{s}$. We consider this question in section 2. Refining and complementing the results of [5], 21], 22] we prove that $W_{s}(D)$ is open, and also dense if not empty. For the similar question related to $\widehat{W}_{s}(D)$ we provide examples to the contrary.

\section{Estimates For $s_{0}(D)$ AND A CharaCterization of the Ball in $\mathbb{C}^{n}$}

1.1. Characterization of the ball by determining sets. This section is devoted to the proof of the following theorem.

Theorem 1.1. Let $D$ be a bounded domain in $\mathbb{C}^{n}$. Then $s_{0}(D)=n+1$ if and only if $D$ is biholomorphic to the unit ball $B^{n}$ in $\mathbb{C}^{n}$.

To verify the estimate $s_{0}\left(B^{n}\right)=n+1$ we need to prove that no $n$ points in $B^{n}$ form a determining set for $A u t\left(B^{n}\right)$. (This was done in [6]; we repeat it here for completeness.) Consider $n$ arbitrary points $\left(p_{0}, p_{1}, \ldots, p_{n-1}\right)$, where $p_{i} \in B^{n}$ for $i=0, \ldots, n-1$. Consider $g \in \operatorname{Aut}\left(B^{n}\right)$ such that $g\left(p_{0}\right)=0$. Now consider $n-1$ vectors $g\left(p_{i}\right)$, and the complex linear space $\pi$ spanned by these vectors. Since $\operatorname{dim}(\pi) \leq n-1$, there is a rotation $f \in \operatorname{Aut}\left(B^{n}\right)$ that is not the identity and keeps all the points of $\pi$ fixed. Now the automorphism $h=g^{-1} \circ f \circ g \in \operatorname{Aut}\left(B^{n}\right)$ is not the identity, and it fixes all $n$ points $\left(p_{0}, p_{1}, \ldots, p_{n-1}\right)$. We proved that $W_{n}\left(B^{n}\right)=\emptyset$, so $s_{0}\left(B^{n}\right)=n+1$.

The rest of this section will be devoted to the proof that $s_{0}(D)=n+1$ implies that $D$ is biholomorphic to the unit ball.

If $H$ is (isomorphic to) a subgroup of the unitary group $U(n)$, let $k(H)$ denote the least number $k$ of vectors $u_{1}, \ldots, u_{k}$ such that if $h \in H$ and if $h\left(u_{j}\right)=u_{j}$ for $j=1, \ldots, k$, then $h=i d$. For $z \in D$ the isotropy group $A u t_{z}(D)$ is isomorphic to the group of its differentials at $z$, and these differentials are unitary with respect to the Bergman inner product on the tangent space $T_{z}(D)$. So $A u t_{z}(D)$ is isomorphic to a subgroup of $U(n)$.

Lemma 1.2. For a bounded domain $D$ in $\mathbb{C}^{n}$,

$$
s_{0}(D) \leq 1+\min \left\{k\left(\text { Aut }_{x}(D)\right): x \in D\right\} .
$$

Proof. Choose $z \in D$ so that $k\left(\operatorname{Aut}_{z}(D)\right)=\min \left\{k\left(A u t_{x}(D)\right): x \in D\right\}$. Denote that number by $k$. Let $u_{1}, \ldots, u_{k}$ be vectors in $T_{z} D$ such that if $h \in \operatorname{Aut}_{z}(D)$ and if $d h(z)\left(u_{j}\right)=u_{j}$ for $j=1, \ldots, k$, then $d h=i d$ (hence $h=i d$ ). For each $u_{j}$, let $z_{j}$ be a point on the geodesic through $z$ in the direction $u_{j}$, so close to $z$ that the geodesic is the unique length minimizing geodesic from $z$ to $z_{j}$. Let $f$ be an automorphism of $D$ fixing $z, z_{1}, \ldots, z_{k}$. Then $d f(z)$ fixes $u_{1}, \ldots, u_{k}$. It follows that $d f(z)=i d$ and $f=i d$. Therefore, $s_{0}(D) \leq 1+\min \left\{k\left(\operatorname{Aut}_{x}(D)\right): x \in D\right\}$. 
Lemma 1.3. If $H$ is a subgroup of $U(2)$ and if $H$ is not transitive on $S^{3}$, then $k(H) \leq 1$.

Proof. Let $S=S^{3}$ be the unit sphere in $\mathbb{C}^{2}$. It suffices to show that the set of fixed points in $S$ of nontrivial elements of $H$ (that is, each of these points is a fixed point of at least one nontrivial element of $H$ ) is not equal to $S$. For $g, h \in U(2)$ and $x \in S, x$ is a fixed point of $h$ iff $g^{-1} x$ is a fixed point of $g^{-1} h g$. So, without any loss of generality we can replace $H$ with a subgroup of $U(2)$ conjugate to $H$.

The Lie algebra $Q$ of $U(2)$ consists of skew Hermitian matrices, so it has as a basis the following elements:

$$
a=\left(\begin{array}{cc}
i & 0 \\
0 & 0
\end{array}\right), \quad b=\left(\begin{array}{cc}
0 & 0 \\
0 & i
\end{array}\right), \quad c=\left(\begin{array}{cc}
0 & -1 \\
1 & 0
\end{array}\right), \quad d=\left(\begin{array}{cc}
0 & i \\
i & 0
\end{array}\right) .
$$

Their Lie bracket relations are

$$
[a, b]=0,[a, c]=-d,[a, d]=c,[b, c]=d,[b, d]=-c,[c, d]=-2 a+2 b .
$$

If $\operatorname{dim} H=4$, then $H=U(2)$, which contradicts the hypothesis that $H$ is not transitive on $S$.

Suppose that $\operatorname{dim} H=3$. One can verify that the only 3 -dimensional Lie subalgebra of $Q$ is spanned by $\{c, d, a-b\}$. Hence, the identity component $H_{0}$ of $H$ is $S U(2)$, again contradicting the hypothesis that $H$ is not transitive on $S$.

Now suppose that $\operatorname{dim} H=2$. One can verify directly that $U(2)$ does not have a subgroup of dimension 2 and rank 1 . Thus, $H$ has rank 2 . Up to conjugation, the identity component $H_{0}$ of $H$ is $T^{2}=\left\{\operatorname{diag}\left(e^{i \alpha}, e^{i \beta}\right): \alpha, \beta \in \mathbb{R}\right\}$. Each component of $H$ is $g T^{2}$. If $h \in g T^{2}$ has a nonzero fixed vector, $h$ must satisfy $\operatorname{det}(h-i d)=0$. It follows that if $\operatorname{dim} H \leq 2$, then the set $U$ of the nontrivial elements of $H$ that have a fixed point on $S$ has dimension $\leq 1$, and for each $g \in U$, the set of fixed points of $g$ on $S$ has dimension 1 . Thus the set $P$ of fixed points of nontrivial elements of $H$ has dimension $\leq 2$. It follows that $P \neq S$. Therefore, $k(H) \leq 1$.

Lemma 1.4. If $H$ is a subgroup of $U(n)$ with $n \geq 2$ and if $H$ is not transitive on $S^{2 n-1}$, then $k(H) \leq n-1$.

Proof. The case where $n=2$ is the previous lemma. Suppose $n>2$ and $H \subset U(n)$ is not transitive on $S^{2 n-1}$. Choose $x, y \in S^{2 n-1}$ so that no element of $H$ maps $x$ to $y$. Choose $z \in S^{2 n-1}$ orthogonal to both $x$ and $y$. Let $S_{1}=\left\{v \in S^{2 n-1}:(v, z)=0\right\}$, where $(v, z)=\sum v_{j} \bar{z}_{j}$, and let $H_{1}=\{g \in H: g(z)=z\}$, and $U_{1}=\{g \in U(n)$ : $g(z)=z\}$. Now $U_{1} \cong U(n-1)$, and $H_{1}$ is a subgroup of $U_{1}$. By the induction hypothesis, since $H_{1}$ is not transitive on $S_{1}, H_{1}$ has a determining set of $n-2$ vectors $\left\{w_{1}, \ldots, w_{n-2}\right\}$. It follows that $\left\{z, w_{1}, \ldots, w_{n-2}\right\}$ is a determining set for $H$. Therefore, $k(H) \leq n-1$.

The proof of our main theorem follows from the following.

Lemma 1.5. If $D$ is a bounded domain in $\mathbb{C}^{n}$, and $D \neq B^{n}$, then $s_{0}(D) \leq n$.

Proof. If $n=1$ the statement is obviously true. Assume $n \geq 2$. Let $z \in D$. Since $D \neq B^{n}, A_{t}(D)$ is not transitive on the directions at $z$, by the main result of $[9]$. By Lemma 1.4, $k\left(\operatorname{Aut}_{z}(D)\right) \leq n-1$. It follows that $s_{0}(D) \leq 1+k\left(\right.$ Aut $\left._{z}(D)\right) \leq$ $n$. 
Remark. For endomorphisms $H(D, D)$ we still have for the ball $\widehat{s}_{0}\left(B^{n}\right)=n+1$. However in the case of endomorphisms there are domains not biholomorphic to the ball but with the same maximum possible value of $\widehat{s}_{0}$. Here are two examples.

For $n=1, \widehat{s}_{0}(D)=\widehat{s}_{0}\left(B^{1}\right)=2$ for any bounded domain $D \subset \mathbb{C}$.

We will now show that for $n=2$, for the unit polydisc $\Delta^{2}, \widehat{s}_{0}\left(\Delta^{2}\right)=\widehat{s}_{0}\left(B^{2}\right)=3$.

Indeed, consider any two distinct points $p_{1}, p_{2} \in \Delta^{2}$. Since the $\operatorname{Aut}\left(\Delta^{2}\right)$ is transitive, we can find an automorphism $g$, such that $g\left(p_{1}\right)=0$. Let $L$ be the complex line through the origin and $g\left(p_{2}\right)$. In terms of the coordinates this line can always be described in one of the forms: $z_{2}=\lambda z_{1}$, or $z_{1}=\lambda z_{2}$, where $|\lambda| \leq 1$. One can check that the map $P:\left(z_{1}, z_{2}\right) \rightarrow\left(z_{1}, \lambda z_{1}\right)$ in the first case or $P:\left(z_{1}, z_{2}\right) \rightarrow$ $\left(\lambda z_{2}, z_{2}\right)$ in the second case will produce a holomorphic retraction of the polydisc, fixing $g\left(p_{1}\right), g\left(p_{2}\right)$. Now the map $g^{-1} P g$ is a holomorphic retraction of $\Delta^{2}$ fixing $p_{1}, p_{2}$. Therefore $\widehat{s}_{0}\left(\Delta^{2}\right)>2$. Since this number is also $\leq 3$, we conclude $\widehat{s}_{0}\left(\Delta^{2}\right)=$ 3 .

1.2. An estimate for $s_{0}(D)$. Let $G$ be a subgroup of $A u t(D)$. By $s_{0}(D, G)$ we denote the minimum number of distinct points in $D$ such that if $g \in G$ and $g$ fixes all these points, then $g=i d$. So, $s_{0}(D)=s_{0}(D, A u t(D))$.

Theorem 1.6. Let $D$ be a bounded domain in $\mathbb{C}^{n}$, let $G$ be a subgroup of $A u t(D)$, and let $q=\operatorname{dim} G$. If $q \geq 1$, then $s_{0}(D, G) \leq q$. If $q=0$, then $s_{0}(D, G) \leq 1$.

Proof. First we consider the case where $q \leq 1$. Let $e$ denote the identity element of $G$, and let $Q=G \backslash\{e\}$. For each $g \in Q$, the set $\{x \in D: g(x)=x\}$ is an analytic set of $D$ of dimension $\leq 2 n-2$. The set $W_{1}:=\{(g, x) \in Q \times D: g(x)=x\}$ is an analytic set of $Q \times D$ of dimension $\leq(2 n-2)+q \leq 2 n-1<\operatorname{dim} D$. Let $W$ denote the set of fixed points of nontrivial elements of $G$. Since $W=\pi\left(W_{1}\right)$, where $\pi: Q \times D \rightarrow D$ is the projection, and since $\operatorname{dim} W_{1}<\operatorname{dim} D$, we see that $W \neq D$. Therefore, $s_{0}(D, G) \leq 1$.

Now we assume that $q \geq 2$. There must be an orbit $Q$ of $G$ of positive dimension. Let $x \in Q$, and let $H:=G_{x}$ be the subgroup of $G$ consisting of elements $g$ satisfying $g(x)=x$. Then $\operatorname{dim} H<\operatorname{dim} G$. By induction hypothesis, $s_{0}(D, H) \leq \operatorname{dim} G-1$. Therefore, $s_{0}(D, G) \leq 1+s_{0}(D, H) \leq \operatorname{dim} G$.

Corollary 1.7. Let $D$ be a bounded domain in $\mathbb{C}^{n}$. If $\operatorname{dim}(\operatorname{Aut}(D)) \geq 1$, then $s_{0}(D) \leq \operatorname{dim}(A u t(D))$. If $\operatorname{dim}(A u t(D))=0$, then $s_{0}(D) \leq 1$.

\section{ON TOPOLOGICAL PROPERTIES OF DETERMINING SETS}

2.1. Determining sets $W_{s}(D)$ are open and dense. Our aim in this section is to prove the following theorem.

Theorem 2.1. Let $D$ be a bounded domain in $\mathbb{C}^{n}$ and $s \geq 1$. Then $W_{s}(D) \subset D^{s}$ is open; if in addition $W_{s}(D) \neq \emptyset$, then $W_{s}(D)$ is dense in $D^{s}$.

The assertion that $W_{s}(D)$ is open and dense in $D^{s}$ was proved for some domains and $s \geq n+1$ in [5]. Using analytic methods of [2], [3, J.P. Vigué (see [21, [22]) proved that $W_{s}(D)$ is open for all bounded domains and all $s$, and that it is dense for $s \geq n+1$. By using the Bergman metric on a bounded domain we are able to use differential geometry methods and the Lie group properties (see [1], 13], [18, also [1], [8], [16]) to prove the above general theorem. 
First we introduce some notation. If $G$ is a subgroup of $\operatorname{Aut}(D), W_{s, G}(D)$ denotes the set of $s$-tuples $\left(x_{1}, \ldots, x_{s}\right)$, where $x_{j} \in D$, such that each element $g \in G$ satisfying $g\left(x_{j}\right)=x_{j}$ for $j=1, \ldots, s$ has to be the identity.

We need the following lemma (Theorem 2.4 in [16]).

Lemma 2.2. Let $\Omega$ be a bounded domain in $\mathbb{C}^{n}$ containing the closure of the unit ball, and $G$ a compact Lie subgroup of Aut $(\Omega)$. Suppose that each $G$-orbit lies in a ball of radius $1 / 2$. Then $G=\{i d\}$.

Lemma 2.3. Suppose that $D$ is a bounded domain in $\mathbb{C}^{n}$ and $G$ is a subgroup of Aut $(D)$. Then $W_{1, G}(D)$ is open in $D$.

Proof. We need to consider only the case $W_{1, G}(D) \neq \emptyset$. Suppose that $x \in W_{1, G}(D)$, and there is a sequence of points in $D, x_{k} \rightarrow x$ such that $x_{k} \notin W_{1, G}(D) \forall k$. Let $U$ be a neighborhood of $x, \bar{U} \subset D$. Then there is a positive $r$ such that for large enough $k$ the ball with the center in $x_{k}$ and of radius $r, b\left(x_{k}, r\right)$ in the Bergman metric compactly lies in $U$. The assertion that $x_{k} \notin W_{1, G}(D)$ means that the subgroup $G_{x_{k}}$ of $G$ fixing $x_{k}$ is not the identity. This subgroup is a compact Lie subgroup of $G$, and also acts on $b\left(x_{k}, r\right)$ (since the Bergman metric is an invariant metric). Applying (a properly adjusted form of) Lemma 2.2, one concludes that there exists an $\varepsilon>0$, such that for large enough $k$ one has an automorphism $g_{k} \in G_{x_{k}}$ and a point $y_{k} \in b\left(x_{k}, r\right) \subset U$ such that the Eucledian distance $\left|g_{k}\left(y_{k}\right)-y_{k}\right|>\varepsilon$. One can now find a subsequence $\left\{k_{j}\right\}$ such that (1) $y_{k_{j}} \rightarrow y \in \bar{U}$, and (2) $g_{k_{j}} \rightarrow g \in G$. We now conclude that $g(x)=x$ and that $|g(y)-y| \geq \varepsilon$. This means that $x \notin W_{1, G}(D)$, which is a contradiction. Therefore $W_{1, G}(D)$ is open in $D$.

Let $\rho(\cdot, \cdot)$ denote the Bergman distance. Let $b(z, r)$ denote the Bergman ball with center $z$ and radius $r$. Let $\bar{b}(z, r)$ be the closure of $b(z, r)$ in $D$.

Lemma 2.4. Suppose that $D$ is a bounded domain in $\mathbb{C}^{n}$ and $G$ is a subgroup of $\operatorname{Aut}(D)$. If $W_{1, G}(D) \neq \emptyset$, then $W_{1, G}(D)$ is dense in $D$.

Proof. In this proof, let $W=W_{1, G}(D)$. Suppose that $W$ is not dense in $D$. Then the closure $K$ of $W$ in $D$ is not equal to $D$. Let $p$ be a boundary point of $K$ in $D$. Choose $r>0$ such that the closure of $b(p, 4 r)$ in $D$ is compact and such that each pair of points of $b(p, 4 r)$ is connected by a unique length-minimizing geodesic segment (in the Bergman metric). There exist points $z, w$ such that $\rho(z, p)<r$, $\rho(w, p)<r, w \in W$, and $z \notin K$. Note that $G(w) \subset W$, where $G(w)$ is the orbit of $w$. Let $Q=G(w) \cap \bar{b}(p, 4 r)$. Then $Q$ is compact and $Q \subset W$. Let $u$ be a point of $Q$ nearest to $z$. Then $u$ is also a point of $G(w)$ nearest to $z$, and $R:=\rho(z, u) \leq \rho(z, w)<2 r$. Choose a point $y$ on the unique length-minimizing geodesic segment from $z$ to $u$ such that $y \notin K$ and $y \neq z$. For each point $x$ of $G(w)$, we see that

$$
\rho(z, y)+\rho(y, x) \geq \rho(z, x) \geq \rho(z, u),
$$

and that the two equalities hold simultaneously only if $x=u$. Hence, $\rho(z, y)+$ $\rho(y, x)>\rho(z, u)=R$ for each $x \in G(w), x \neq u$. It follows that $\rho(y, x)>R-$ $\rho(z, y)=\rho(y, u)$ for each $x \in G(w), x \neq u$. Therefore, $u$ is the unique point of $G(w)$ nearest to $y$. Since $y \notin K$, there is a nontrivial $g \in G$ such that $g(y)=y$. Now $\rho(y, u)=\rho(g(y), g(u))=\rho(y, g(u))$ forces $g(u)=u$. Since $u \in W$, the map $g$ must be the identity, contradicting the fact that $g$ is not trivial. Therefore, $W_{1, G}(D)$ is dense in $D$. 
Proof of Theorem 2.1. We need to prove this theorem only for $W_{s}(D) \neq \emptyset$. For $g \in \operatorname{Aut}(D)$ let $Q_{s}(g)$ denote the mapping

$$
Q_{s}(g): D^{s} \rightarrow D^{s}, \quad Q_{s}(g)\left(z_{1}, \ldots, z_{s}\right)=\left(g\left(z_{1}\right), \ldots, g\left(z_{s}\right)\right) .
$$

Let $G=\left\{Q_{s}(g): g \in \operatorname{Aut}(D)\right\}$. Then $G \subset \operatorname{Aut}\left(D^{s}\right)$, and $W_{1, G}\left(D^{s}\right)=W_{s}(D)$. By the previous lemmas, $W_{s}(D)$ is open and dense in $D^{s}$.

2.2. Determining sets $\widehat{W}_{s}(D)$ that are not open. In 22] it was proved that $\widehat{W}_{s}(D)$ is open in $D^{s}$ for any bounded taut domain in $\mathbb{C}^{n}$. Our aim in this section is to present an example in $\mathbb{C}^{2}$ of a bounded domain such that the determining set $\widehat{W}_{2}(D)$ is not open in $D^{2}$.

First we construct the set $D \subset \mathbb{C}^{2}$.

Denote $B_{2}=\left\{z \in \mathbb{C}^{2}|| z \mid<2\right\}, B_{1}=\left\{z \in \mathbb{C}^{2}|| z \mid<1\right\}, b_{1}=\left\{z=\left(z_{1}, z_{2}\right) \in\right.$ $\left.\left.\mathbb{C}^{2}|| z_{1}\right|^{2}+\left|z_{2}+1\right|^{2}<\left(1-10^{-4}\right)^{2}\right\}, b_{2}=\left\{z=\left(z_{1}, z_{2}\right) \in \mathbb{C}^{2}|| z_{1}-\left.0.02\right|^{2}+\left|z_{2}+1\right|^{2}<1\right\}$. Let $\Omega=\left(B_{2} \backslash \overline{B_{1}}\right) \cup b_{1} \cup\left(b_{2} \cap B_{2}\right)$.

Now consider pairs of points $p_{j}, q_{j} \in \Omega, p_{j}=\left(-1.5,2^{-j}\right), q_{j}=\left(1.5,2^{-j}\right)$, and bydiscs $U_{j}=\left\{z=\left(z_{1}, z_{2}\right) \in \mathbb{C}^{2}|| z_{1}|<1.5,| z_{2}-2^{-j} \mid<2^{-2^{j}}\right\}, j=1,2, \ldots$. Also, finally domain $D=\Omega \cup \bigcup_{j=1}^{\infty} U_{j}$.

Note the following properties:

1. $D$ is a connected domain, $D \subset B_{2}$.

2. The entire complex disc $\Delta_{j}=\left\{z=\left(z_{1}, z_{2}\right) \in B_{2} \mid z_{2}=2^{-j}\right\} \subset D$ for all $j$.

3. $\lim _{j \rightarrow \infty} p_{j}=p_{0}=(-1.5,0), \lim _{j \rightarrow \infty} q_{j}=q_{0}=(1.5,0)$, and for the disc $\Delta_{0}=\left\{z=\left(z_{1}, z_{2}\right) \in B_{2} \mid z_{2}=0\right\}, \Delta_{0} \cap D=\left\{z \in \mathbb{C}^{2}|1<| z_{1} \mid<2, z_{2}=0\right)$.

4. $U_{i} \cap U_{j}=\emptyset$ for $i \neq j$ for large enough $i, j$.

5. Let $l_{0}=\left(0,-10^{-4}\right) \in \overline{b_{1}}$ be the "tip" of this ball, and the point of $\overline{b_{1}}$ closest to the origin. Let $d$ denote the Kobayashi distance in $B_{2}$ from the origin to $l_{0}$, and $\bar{k}=\bar{k}(0, d)$ the closed Kobayashi ball with center at the origin and radius $d$. Then $\bar{k} \cap \overline{\left(b_{1} \cup b_{2}\right)}=\left\{l_{0}\right\}$.

We are going to show that for any $j$ the pair $\left(p_{j}, q_{j}\right) \notin \widehat{W}_{2}(D)$, but their limit $\left(p_{0}, q_{0}\right) \in \widehat{W}_{2}(D)$, which will prove that $\widehat{W}_{2}(D)$ is not open in $D^{2}$.

Statement 1. For any $j$, there is a holomorphic retraction of $B_{2}$ (and therefore of $\left.D \subset B_{2}\right)$ onto $\Delta_{j}$, and since $\left(p_{j}, q_{j}\right) \in \Delta_{j}$, the pair $\left(p_{j}, q_{j}\right) \notin \widehat{W}_{2}(D)$.

To prove this one needs to first use an automorphism $g$ of $B_{2}$ to move $\Delta_{j}$ to $\Delta_{0}$, use the natural projection $P$ of the ball $B_{2}$ onto $\Delta_{0}$, and set the needed holomorphic retraction as $g^{-1} \circ P \circ g$.

Statement 2. Any holomorphic map $f: D \rightarrow D$ extends to a holomorphic map $F: B_{2} \rightarrow B_{2}$.

Statement 3. Any holomorphic map $F: B_{2} \rightarrow B_{2}$ that fixes our two points $p_{0}, q_{0}$ will fix all the points of $\Delta_{0}$.

For proof see (22, ex. 1 in sec 4$)$.

Now let $f: D \rightarrow D$ be a holomorphic map that fixes our two points $p_{0}, q_{0}$. Its extension $F: B_{2} \rightarrow B_{2}$ will be an identity on $\Delta_{0}$.

Statement 4. $F\left(l_{0}\right)=l_{0}$.

Indeed, consider $K$ the Kobayashi ball in $B_{2}$ with center at the origin that coincides with the standard unit ball in $\mathbb{C}^{2} . K \cap D$ consists of nonintersecting connected pieces, only one of which, namely $G=K \cap\left(b_{1} \cup b_{2}\right)$, has a point $(0.02,0)$ on $\Delta_{0}$ as 
a limit point. Since this point is fixed by $F$, and $F$ cannot increase the Kobayashi distance, we conclude that $F(G) \subseteq G$.

Since it is also true that $F(\bar{k}) \subseteq \bar{k}$, the only possible image for $l_{0}$ under $F$ is the point itself (see property 5 above), so $F\left(l_{0}\right)=l_{0}$.

We now conclude our observation by pointing out that $F: B_{2} \rightarrow B_{2}$ has three fixed points, $p_{0}, q_{0}, l_{0}$. By ([22, ex. 1 in sec 4$) F$ is the identity, so $f: D \rightarrow D$ is the identity, and therefore $\left(p_{0}, q_{0}\right) \in \widehat{W}_{2}(D)$.

2.3. Determining sets $\widehat{W}_{s}(D)$ that are not dense. Our goal here is to present an example of a domain $D$ such that for any $s$ the determining set $\widehat{W}_{s}(D)$ is not dense in $D^{s}$.

J.-P. Vigué (see 22, ex. 2 in sec 4) has provided such an example for $D=$ $\Delta^{2}=\left\{z=\left(z_{1}, z_{2}\right) \in \mathbb{C}^{2}|| z_{1}|<1,| z_{2} \mid<1\right\}$, the polydisc in $\mathbb{C}^{2}$, and $s=3$. For completeness, using the same idea, here we provide an example for $\Delta^{2}$ and any $s \geq 3$.

Fix $s$ points $\left(A_{j}, 0\right), A_{j}=2^{-j}, j=1, \ldots, s$. The set $T$ of these points is a point in $\left(\Delta^{2}\right)^{s}$. T $T \notin \widehat{W}_{s}\left(\Delta^{2}\right)$, since $\left(z_{1}, z_{2}\right) \mapsto\left(z_{1}, 0\right)$ is a holomorphic retraction of $\Delta^{2}$, fixing all these points. Let $\widetilde{T}=\left\{\left(a_{j}, b_{j}\right), j=1, \ldots, s\right\} \in\left(\Delta^{2}\right)^{s}$ be any $\delta>0$ perturbation of $T$. So, $\sum_{j=1}^{s}\left(\left|A_{j}-a_{j}\right|^{2}+\left|b_{j}\right|^{2}\right)<\delta^{2}$. We will show that if $\delta$ is small enough, $\widetilde{T} \notin \widehat{W}_{s}\left(\Delta^{2}\right)$.

Consider the Lagrange interpolation polynomial $\varphi(w)=\sum_{j=1}^{s} b_{j} \prod_{i \neq j} \frac{\left(w-a_{i}\right)}{\left(a_{j}-a_{i}\right)}$. One can verify that if $\delta>0$ is small enough ( $\operatorname{say} \delta<4^{-s^{2}}$ ), then $|\varphi(w)|<1$ if $|w|<1$. Now the map $\left(z_{1}, z_{2}\right) \mapsto\left(z_{1}, \varphi\left(z_{1}\right)\right)$ is a holomorphic retraction of the unit polydisc that has $\widetilde{T}$ in its set of fixed points. Therefore $\widetilde{T} \notin \widehat{W}_{s}\left(\Delta^{2}\right)$.

As a remark we note that by using this idea one can construct many such examples. Moreover the following theorem holds.

Theorem 2.5. Consider the topological space $\check{D}_{n}$ of all bounded domains in $\mathbb{C}^{n}$ with the topology induced by the Hausdorff distance between boundaries of the domains. Let $\breve{G}_{n} \subset \check{D}_{n}$ be such that if $D \in \breve{G}_{n}$, then $\widehat{W}_{s}(D)$ is not dense in $D^{s}$ for all $s \geq 1$. Then $\breve{G}_{n}$ is dense in the topological space $\check{D}_{n}$.

\section{REFERENCES}

[1] E. Bedford and J. Dadok, Bounded domains with prescribed group of automorphisms, Comment. Math. Helv., 62 (1987), 561-572. MR0920057 (89c:32078)

[2] H. Cartan, Les fonctions de deux variables complexeses et le problème de la représentation analytique, J. Math. pures et appl., $9^{e}$ série, 11 (1931) 1-114.

[3] H. Cartan, Sur les fonctions de plusieurs variables complexes. L'itération des transformations intérieures d'un domaine borné, Math. Z., 35 (1932) 760-773. MR.1545327

[4] S. D. Fisher and John Franks, The fixed points of an analytic self-mapping, Proc. AMS, 99 (1987), 76-78. MR0866433 (87m:30069)

[5] B. L. Fridman, K. T. Kim, S. G. Krantz, \& D. Ma, On fixed points and determining sets for holomorphic automorphisms, Michigan Math. J., 50 (2002), 507-515. MR1935150 (2003i:32034)

[6] B. L. Fridman, K. T. Kim, S. G. Krantz, \& D. Ma, On determining sets for holomorphic automorphisms, to appear in Rocky Mountain J. of Math.

[7] B. L. Fridman and E. A. Poletsky, Upper semicontinuity of automorphism groups, Math. Ann., 299 (1994), 615-628. MR1286888 (96b:32040) 
[8] B. L. Fridman, D. Ma, E. A. Poletsky, Upper semicontinuity of the dimensions of automorphism groups of domains in $\mathbb{C}^{n}$, Amer. J. Math., 125 (2003), 289-299. MR.1963686 (2004f:32027)

[9] R. E. Greene and S. G. Krantz, Characterization of complex manifolds by the isotropy subgroups of their automorphism groups, Indiana Univ. Math. J., 34 (1985), no. 4, 865879. MR0808832 (87b:32061)

[10] R. E. Greene and H. Wu, Function theory on manifolds which possess a pole, Lecture Notes in Mathematics, 699, Springer, Berlin, 1979. MR0521983 (81a:53002)

[11] D. Gromoll, W. Klingenberg, and W. Meyer, Riemannsche Geometrie im Grossen, $2^{\text {nd }}$ ed., Lecture Notes in Mathematics, v. 55, Springer-Verlag, New York, 1975. MR0365399 (51:1651)

[12] K. T. Kim, S. G. Krantz, Determining sets and fixed points for holomorphic endomorphisms, Contemporary Math. 328 (2003), 239-246. MR1990405 (2004e:32012)

[13] W. Klingenberg, Riemannian Geometry, $2^{\text {nd }}$ ed., de Gruyter Studies in Mathematics, Berlin, 1995. MR 1330918 (95m:53003)

[14] S. Kobayashi, Hyperbolic Complex Spaces, Springer, 1999. MR1635983 (99m:32026)

[15] K. Leschinger, Über fixpunkte holomorpher Automorphismen, Manuscripta Math., 25 (1978), 391-396. MR0509592 (80e:55005)

[16] D. Ma, Upper semicontinuity of Isotropy and automorphism groups, Math. Ann., 292 (1992), 533-545. MR1152949 (92m:32055)

[17] B. Maskit, The conformal group of a plane domain, Amer. J. Math., 90 (1968), 718-722. MR 0239078 (39:437)

[18] D. Montgomery, L. Zippin, Topological transformation groups, Interscience, New York, 1955. MR0073104 (17:383b)

[19] E. Peschl and M. Lehtinen, A conformal self-map which fixes 3 points is the identity, Ann. Acad. Sci. Fenn., Ser. A I Math., 4 (1979), no. 1, 85-86. MR0538091(80h:30010)

[20] N. Suita, On fixed points of conformal self-mappings, Hokkaido Math. J., 10 (1981), 667671. MR0662329 (83f:30006)

[21] J.-P. Vigué, Sur les ensembles d'unicité pour les automorphismes analytiques d'un domaine borné, C. R. Acad. Sci. Paris, Ser. I 336 (2003), 589-592. MR.1981474 (2004b:32028)

[22] J.-P. Vigué, Ensembles d'unicité pour les automorphismes et les endomorphismes analytiques d'un domaine borné, Annales Institut Fourier, 55 (2005), 147-159. MR2141692 (2006b:32023)

Department of Mathematics, Wichita State University, Wichita, Kansas 67260-0033

E-mail address: buma.fridman@wichita.edu

Department of Mathematics, Wichita State University, Wichita, Kansas 67260-0033

E-mail address: dma@math.wichita.edu 